\title{
Statement of Principles on the Return of Research Results and \\ Incidental Findings in Paediatric Research: A Multi-site Consultative Process
}

\begin{tabular}{|r|l|}
\hline Journal: & Genome \\
\hline Manuscript ID & gen-2015-0092.R1 \\
\hline Manuscript Type: & Article \\
\hline Date Submitted by the Author: & 09-Oct-2015 \\
\hline Complete List of Authors: & $\begin{array}{l}\text { Sénécal, Karine; Centre of Genomics and Policy, McGill University, Human } \\
\text { Genetics } \\
\text { Rahimzadeh, Vasiliki; Centre of Genomics and Policy, McGill University, } \\
\text { Human Genetics } \\
\text { Knoppers, Bartha; Centre of Genomics and Policy, McGill University, } \\
\text { Human Genetics } \\
\text { Fernandez, Conrad; IWK Health Centre, Department of Pediatrics, } \\
\text { Dalhousie University, } \\
\text { Avard, Denise; Centre of Genomics and Policy, McGill University, Human } \\
\text { Genetics } \\
\text { Sinnett, Daniel; Sainte-Justine University Health Center, Research Center, }\end{array}$ \\
\hline Keyword: & $\begin{array}{l}\text { genetic/genomic research, paediatrics, return of results, incidental findings, } \\
\text { ethics }\end{array}$ \\
\hline \multicolumn{2}{|l}{} \\
\hline
\end{tabular}




\section{Statement of Principles on the Return of Research Results and Incidental Findings in Paediatric Research: A Multi-site Consultative Process}

Karine Sénécal $^{\mathrm{a}}$, Vasiliki Rahimzadeh ${ }^{\mathrm{b}}$ Bartha M Knoppers $^{\mathrm{c}}$, Conrad V Fernandez ${ }^{\mathrm{d}}$, Denise Avard $^{\mathrm{e}}$, Daniel Sinnett ${ }^{\mathrm{f}}$

${ }^{a}$ LL.M. Academic Associate, Centre of Genomics and Policy (CGP), Faculty of Medicine, Department of Human Genetics, McGill University, 740, avenue Dr. Penfield, \#5202 Montreal (Quebec), Canada H3A 0G1

${ }^{\mathrm{b}} \mathrm{PhD}$ candidate, Research Assistant, Centre of Genomics and Policy (CGP), Faculty of Medicine, Department of Family Medicine, McGill University,740, avenue Dr. Penfield, \#5203, Montreal (Quebec), Canada H3A 0G1

${ }^{\mathrm{c}} \mathrm{Ph}$.D. Director Centre of Genomics and Policy, Faculty of Medicine, Department of Human Genetics, McGill University, Canada Research Chair in Law and Medicine, 740, avenue Dr. Penfield, \#5214

Montreal (Quebec), Canada H3A 0G1

${ }^{\mathrm{d}}$ MD, Professor, IWK Health Centre, Department of Pediatrics, Dalhousie University, 5850 University Avenue, P. O. Box 9700

Halifax, Nova Scotia, Canada B3K 6R8

${ }^{\mathrm{e}} \mathrm{Ph}$.D. Research Director, Centre of Genomics and Policy, Faculty of Medicine, Department of Human Genetics, McGill University, and associate professor at the Faculty of Medicine, Department of Human Genetics at McGill University, 740, avenue Dr. Penfield, \#5210 Montreal (Quebec), Canada H3A 0G1

${ }^{\mathrm{f}}$ Ph.D. Senior Scientist, Sainte-Justine University Health Center, Research Center, Full Professor Department of Pediatrics, University of Montreal, 3175 Chemin de la Côte Sainte-Catherine, Montreal, Québec, Canada H3T 1C5 


\begin{abstract}
This paper proposes a set of recommendations for the return of research results and incidental findings in paediatrics. The Québec Network of Applied Genetic Medicine spearheaded the initiative to develop the Statement of Principles on the Return of Research Results and Incidental Findings, which was the result of a consultation process with clinical and research experts in the field. To formulate the Statement of Principles, the authors: (1) reviewed empirical and grey literature on the return of research results and incidental findings in Europe and Canada; (2) conducted a qualitative study of stakeholder groups; (3) developed and (4) validated the recommendations through consultations with the stakeholder groups. The Statement of Principles provides a useful disclosure tool for deciding when, and under what circumstances to return research results and incidental findings. It addresses the issue of return of results in genetic research generally, and has also specific principles for various research contexts, including paediatric research. It delineates ethical issues unique to paediatric research, and provides a framework to guide research ethics committees as well as the research community in addressing these issues.
\end{abstract}

KEYWORDS: genetic/genomic research, paediatrics, return of results, incidental findings, ethics.

\title{
INTRODUCTION
}

Rapid advances in research and clinical sequencing underscores the immediacy of national and international consensus regarding the return of research results and incidental findings. Although a number of professional bodies both in Canada (Boycott et al. 2015) and the United States (Green et al. 2013) have proposed guidelines outlining ethical terms of disclosure, 
they are primarily intended for application in clinical contexts. Return of results and incidental findings discovered during the course of research has not, in contrast, received similar policy attention. This is particularly true for clinical genetic research involving children (Green et al. 2013; McGuire et al. 2013; Wolf et al. 2013; Abdul-Karim et al. 2013) where ethical considerations may be more acute.

In its 2010 revision, the Tri-Council Policy Statement: Ethical Conduct for Research Involving Humans (Canadian Institutes of Health Research, Natural Sciences and Engineering Research Council of Canada, and Social Sciences and Humanities Research Council of Canada 2010)(hereafter referred to as TCPS2) mandated Canadian researchers and research ethics boards (REBs) to ensure protocols incorporate a plan for managing research results, and obligated them to disclose any "material" incidental findings to participants. Although the TCPS2 established such obligations, it did not specifically address the process of how this disclosure should occur. Nor did it take into consideration the local specificities of diverse research contexts. Although the most recent 2014 version introduced some exceptions to disclosure, it added little clarity for researchers and REBs on how to ensure such disclosure respects patients and their families (Canadian Institutes of Health Research Natural Sciences and Engineering Research Council of Canada and Social Sciences and Humanities Research Council of Canada 2014).

This paper details the consultation process and resultant guidelines intended to fill this policy gap. The authors, in collaboration with clinical and research experts developed the Quebec Network of Applied Genetic Medicine Statement of Principles on the Return of Research Results and Incidental Findings (hereafter RMGA Statement). The RMGA Statement is available online in English and French (Quebec Network of Applied Genetic Medicine) and provides tools for researchers and REBs members to plan for the management of information; to make decisions about results that should/could be returned (or not); and how, when, to whom, and by whom the 
results should be returned. Although the return of research results and incidental findings raises general challenges in most genetic research contexts, the RMGA Statement also includes recommendations for child-specific issues, intra-familial communication, biobank research and longitudinal studies. We present the paediatric-specific recommendations in this article, and detail the consultation process used to develop the RMGA Statement.

\section{Whole genome sequencing in paediatrics: policy and applications}

Genome sequencing, both whole exome (WES) and whole genome (WGS), generates information that may or may not be directly related to the research objectives. Information not related to primary research questions is considered incidental, but may have clinical significance for the health status of the child either in their immediate future or in adulthood. The majority of incidental findings, however, lack adequate clinical validity and utility (Wright et al. 2011; Bredenoord et al. 2011). Moreover, incidental findings may be relevant not only for the child but also for parents, siblings and other biological relatives.

Genome sequencing in a research context therefore brings to the fore many questions ranging from concerns about privacy and discrimination; the psychological burden of adverse genomic findings; challenges of informed consent; implementation of pre and post test counselling (Netzer et al. 2009; Fabsitz et al. 2010); the possible duty to actively search for Incidental Findings (IF) (Green et al. 2013); and the nature of the duty to inform, to say nothing of the means, scope and duration of such responsibilities for healthcare professionals and researchers (Burke et al. 2013; Clayton \& McGuire 2012). These issues can be accentuated in the paediatric context where a child's inability to consent and clinical implications for their future health weigh heavily in considerations to disclose (or not). 
Projects to pilot the first genomic sequencing clinics for children are already underway, as research project, in some children's hospitals in Canada (Sainte Justine UHC), underlining the timeliness of the proposed RMGA Statement and paediatric-specific recommendations. In response to the challenges of IF disclosure in paediatric research, some commentators argue the disclosure of medically significant IF i.e. preventable, adult-onset conditions should not be limited by the age, and should be shared with parents or family member(s) (Green et al. 2013; Evans 2013; Beskow \& Burke 2010). Others argue the obligation to inform may become more pressing commensurate with the nature of the condition under study, continuity of care, the dual role some health care professionals assume as researchers, and the source of the samples donated to research (e.g. biobanks) (Evans 2013). Irrespective of these factors, studies demonstrate that research participants generally express a "strong right" to receive genetic research results even if the findings do not directly relate to the target research question (Beskow \& Burke 2010; Fernandez et al. 2013).

As genomic sequencing becomes a centerpiece in paediatric research (B. Knoppers et al. 2014), there is an urgent need for guidance on how to address the return of individual results and IF to children and their families (Fernandez \& Canadian Paediatric Society Bioethics Committee 2008; B. M. Knoppers et al. 2014). While written and approved for researchers in the province of Quebec, the principles articulated herein are relevant and valuable to all engaged in paediatric genomic research across Canada and internationally.

\section{METHODS}

To prepare the recommendations outlined in the RMGA Statement, we: i) systematically reviewed empirical and grey literature; ii) conducted a qualitative descriptive study with 
participants and researchers exploring their perceptions on the return of paediatric genomic research results; iii) drafted recommendations for the paediatric research contextí and iv) validated the recommendations through consultations with RMGA members as well as with partners in the research community (the Finding of Rare Diseases Genes in Canada Consortium (hereafter FORGE Canada Consortium), the Mother, Infant, Children, Youth, Research Network (hereafter MICYRN). Each phase of consultation process is described below.

\section{i) Literature and Policy Review}

We examined ethical, legal and social issues relevant to WGS, IF and paediatric research. A total of 40 documents were retrieved in English and French using Web of Science. Queries were limited to 2005-2014 and included the following search terms: [topic]: (ethics) or (legal) or (bioethics) AND [title]: (incidental findings) or (unsolicited findings) or (research results) AND [topic]: (pediatric] or (paediatric) or (child) or (children) or (minors). The international and national policies retrieved and referenced in this text were collected using a snowball method from the following two sources to ensure the capture of all relevant documents: (i) HumGen International Database (http://www.humgen.org), a database of international, national, and regional guidelines and policies specific to human genetic research (Avard et al. 2011); and (ii) websites of international, national and regional professional organizations. Our reviews (Sénécal et al. 2015; Fernandez et al. 2014) corroborated the lack of consensus and specific guidance on communicating IF discovered in paediatric research.

\section{ii) Qualitative and Quantitative Studies of Stakeholder Groups}

The authors conducted qualitative and quantitative studies with parents of children involved in paediatric research and genomic researchers from four large-scale projects: Canadian Pediatric Cancer Genome Consortium (hereafter CPCGC), Orphan Diseases: Identifying Genes 
and Novel Therapeutics to Enhance Treatment (hereafter IGNITE), the FORGE Canada Consortium as well as the Terry Fox New Frontiers Program Project in Genomic determinants of childhood leukemia (Kleiderman et al. 2014; Fernandez et al. 2013). We found the majority of parents preferred disclosure of findings relevant to the target of the genomic research and any IF (Fernandez et al. 2014). Independent of the research context and clinical significance of the result, parents felt they should be made aware of all results. Parents asserted that they alone were responsible for transmitting important genetic information to their child. Despite the potential for harm, parents perceived the benefits of receiving IF to outweigh the risks (Kleiderman et al. 2014). A thorough analysis of the feasibility for managing IF in the research context must take into account the cost associated with the communication of such results, the impact on the health care system, as well the practical realities researchers face with respect to limited funding, lack of clinical expertise and the volume of research data. Researchers felt, for the most part, an obligation to offer targeted and incidental results if identified, but felt less strongly that IF needed to be actively pursued (Fernandez et al. 2013). Emerging from research with clinicians and clinical researchers was thus a continuum of decisions to return research results depending on numerous contextual factors, including the best interests of children, the clinical significance of the findings and the need to adhere to professional standards (Rahimzadeh et al. 2014). Given the multitude of viewpoints on this issue, an ethically and socially responsible approach is needed for the return of research results and incidental findings in paediatric genomic research.

\section{iii) Drafting the Statement of Principles on the Return of Research Results and Incidental}

\section{Findings}

A multidisciplinary working group from the Centre of Genomics and Policy-comprised of members with expertise in law and bioethics related to genomic research, paediatric research, and biobanks - developed a draft of the RMGA Statement. The draft synthesized pertinent 
ethical, legal and social issues emerging from the literature, policy analyses and the empirical research noted above. The working group presented the first draft of the Statement to the RMGA in Montreal, Quebec in May of 2012. Based on comments from this workshop, a revised Statement was e-mailed to over 100 members of the RMGA who were invited to provide further written comments. We received constructive comments from a dozen members. The working group integrated these comments into a second draft, which was then circulated for international commentary.

\section{iv) Validated the Draft Statement of Principles with Various Stakeholders}

We consulted with numerous national and international stakeholders including the i) $\mathrm{P}^{3} \mathrm{G}$ International Paediatric Platform, composed of international experts in the ethics of paediatric genomics (Programmes, Public Population Projects in Genomics and Society); ii) legal scholars in the United States with expertise in policy issues in genomics research and paediatrics, and who reviewed the applicable legal rules for returning results (Clayton 2013); iii) members of consumer groups such as the Canadian Organization of Rare Diseases (CORD) (https://www.raredisorders.ca/about-cord/) and the Quebec Coalition of Orphan Diseases (Regroupement québécois des maladies orphelines (RQMO) (http://rqmo.org/wp/); and iv) leaders of the CPCGC, IGNITE, and the MICYRN, a Canadian federally incorporated non-profit society of 19 maternal child health research institutions. The Working Group presented the final draft to the RMGA Board of Directors and the RMGA Statement was officially adopted in May 2013.

\section{RMGA Statement for paediatric research:}


Box 1 presents the paediatric-specific recommendations included in the RMGA Statement. The recommendations provide justification and rationale for reporting individual results and IF in paediatrics, reasons to refrain from disclosure, and procedures for handling individual results and IF. Recommendations and supporting rationale are described in further detail below.

BOX 1. RMGA Recommendations for Reporting Individual and Incidental Findings in Paediatric Research

\section{RECOMMENDATION}

i. Individual results and incidental findings concerning the child MUST be returned when:

ii. Individual results and incidental findings should generally $\underline{\text { NOT be }}$ returned when they concern the future health of the minor or predisposition to late-onset disorders*.

*However, results and findings may be offered to the minor's parent under the following exceptional circumstances if these criteria are met:

iii. Procedures for handling individual results and incidental findings should include:

\section{CRITERIA}

- Results meet the generally accepted criteria of scientific and clinical validity;

- Results have significant implications for the health of the minor;

- Effective treatment or prevention is available and should be initiated during childhood or adolescence;

- REB approval has been obtained; and

- The research result has been confirmed by an accredited clinical diagnostic laboratory

- Results meet the generally accepted criteria for scientific and clinical validity;

- Results are important or the immediate health of a parent or adult-aged sibling, considering:

$\rightarrow$ the probability of the risk occurring (for the parents or adult-aged sibling) is high;

$\rightarrow$ the consequences for the parent's or adult-aged sibling are serious;

$\rightarrow$ treatment or an effective method of prevention is available;

- REB approval has been obtained;

- The research result has been confirmed by an accredited clinical diagnostic laboratory before return;

- Parental consent has been obtained; and

- When possible and appropriate, the assent of the adolescent has been obtained

- Management of information

- Who should return the results?

- How should the results be communicated?

- When should the results be communicated? 
- To whom should the results be communicated?

\section{i) Must be Returned}

The predominant ethical arguments supporting the disclosure of individual target results and IF in paediatrics are focused on safeguarding the best interests of the child (United Nations Convention on the Rights of the Child 1989). These research results may have immediate impact on the health of the child, or may be relevant to their future health. We recommend that parents cannot refuse return of results or IF when all criteria are met, as the child is likely to benefit clinically from receiving this information. For example, discovering a serious early-onset disease which, according to evidence-based medicine, is amenable to treatment or prevention during childhood or adolescence should be returned to parents or legal guardians. Parental refusal in this regard could be considered negligence, as it would impede the delivery of appropriate care necessary to ensure the health of their child. The researcher should consult with an ethics committee and refer to child services if appropriate.

The authors recognize that researchers may not have the expertise to convey these genomic findings to participants directly, or have fostered a clinical relationship in order to do so. As such, disclosure may be most appropriate through a third party health care provider.

\section{ii) Should Not Be Returned}

We recommend individual genomic results and IF concerning the future adult health of a minor should not be returned unless a clinical intervention should be initiated during childhood. Testing for adult-onset conditions and communication of such results should be deferred until adulthood when the child can provide fully informed consent except as noted below. Disclosing 
such findings could engender more harm than good and could compromise the child's best interest. Possible harms as a result of disclosure include heightened anxiety among parents, differential treatment of children based on unfavourable results and detrimental psychosocial effects. In addition, the provision of results may infringe on the child's "right to an open future" (Davis 2009) i.e. potentially violating his or her right to make health decisions regarding personal genetic information.

We also do not oblige a researcher to re-contact child participants with the intent to offer individual research results once they become adults. Differences in legal and ethical obligations between researchers and clinicians - namely the former's lack of a fiduciary duty to patientssubstantiate this position. The researcher-participant relationship in this regard should terminate at the completion of the study. This policy should hold regardless of the research design, including longitudinal studies in which the research project may be completed prior to adulthood. Mandating participant re-contact would place unfeasible logistical and financial burdens on researchers and could impede future research efforts.

Consequently, if participants reach the age of majority during the course of the

research, the researchers should offer the results or IF to participants if the results meet the proposed criteria (i.e. scientific and clinical validity; clinical utility; REB approval; consent of the participant).

- Exceptions to delayed return of adult onset findings

Under certain exceptional circumstances, it is possible that disclosure of significant and validated genetic results can be directly beneficial to the child in preserving the life of loved ones or caregivers. One example would be the discovery of a minor's carrier status for a BRCA1 or BRCA2 mutation of known clinical significance. Knowing this information may not immediately 
impact the child's health, however the information may be relevant to the health of the child's parent(s) or adult siblings. As the BRCA example demonstrates, communicating such results can have significant consequences for the child's biological relatives, for whom the success of preventive interventions and ongoing surveillance depends on timely disclosure. Improving parental or familial health outcomes may therefore justify the psycho-social risk and loss of future autonomy.

We caution against a "one size fits all" approach, however, and advise on maintaining flexibility when evaluating the situation on a case-by-case basis. Such situations should be considered together with the researcher and the research ethics committee. If deemed appropriate to disclose IF, pre-established procedures during the consent process should be followed. If there are no established procedures, researchers should, at a minimum, respect the individual's voluntary receipt of the information and plan to answer questions or provide referrals for clinical counselling.

\section{iii) Procedures for Handling Individual Results and Incidental Findings}

The researcher should anticipate the kinds of research results and findings that could possibly emerge in the course of the study, as well plan for disclosure procedures in their wake (Canadian Institutes of Health Research Natural Sciences and Engineering Research Council of Canada and Social Sciences and Humanities Research Council of Canada 2014). The minor's legal representatives should be informed of this disclosure plan for such results or IF as much as can be reasonably anticipated at the time of the consent process. Where applicable, the researchers should seek parental preferences and adolescent assent regarding disclosure of anticipated results, and this plan should be approved by an REB.

\section{- Management of information}


The conditions and manner of disclosure should be made clear to participants and families. The researchers should outline steps for managing IF and explain the disclosure policy for each of the following:

1) individual results and IF important to the immediate health of the child and meet the criteria for mandatory return of results by a researcher or qualified health care professional (refer to Box 1);

2) individual results and IF that reveal information about the future health of the child for which there is no treatment or preventive measure in childhood or adolescence and meet the criteria for non-communication; and

3) results concerning the future health of the minor and meet criteria for disclosure under exceptional circumstances, such as those implicating the health of the parents or siblings and for which there is an effective intervention. The plan for managing information should present options and seek parental preferences.

- Who should return the results?

Researchers can return results to the parents of child participants if they have the necessary professional competency to do so, and refer them to appropriate resources. If the researcher does not have the necessary professional competency to return results and explain their implications, then appropriate plans should be in place for timely clinical referral (e.g. to a physician, nurse or genetic counsellor). Additionally, communication modalities should be clearly outlined during the consent process. Researchers should encourage sharing these findings with the family's primary health care providers so they can use this information to fully contextualize the care of the child.

It is impossible to apply a single approach to all research contexts. Discoveries made during the course of secondary research using biological materials from biobanks illustrate this. 
A secondary researcher would rarely be responsible for sharing results with the participant. Rather, biobank policies should identify a responsible party for communicating results. The terms of a researcher's responsibilities should be incorporated in any agreement for the transfer of medical/genetic data or biological material.

- How should the results be communicated?

Researchers and/or relevant experts (e.g. genetic counsellor, pediatrician) should provide the parents (or the child, if appropriate to their age and maturity) with clear explanations in lay terms, and be prepared to address any possible questions. Researchers should also address the following elements:

- Possible preventative options or treatment

- The limits of predicting the consequences of the genetic finding

- The possible limits of clinical services/resources for the condition

- The broad consequences of the results for the participant's family, and

- The accessibility of genetic counselling services.

In case of doubt concerning the disclosure method, researchers should consult with their REB or colleagues.

Researchers who envision returning individual results or IF in genetic research should engage genetic counselling services, where pertinent. They are often better able to explain the clinical significance or consequences of the genetic information, such as the prospective implications of the findings for the participant's family members. Only trained and experienced professionals should offer genetic counselling.

- When should the results be communicated?

Timing the disclosure of individual results and IF depends on the type of results, the clinical and familial context. In all cases, individual results and IF should be returned - after the confirmation of the result by an accredited clinical diagnostic laboratory - as soon as possible in the research process, and at the very least concurrent with scientific publication. Contingencies 
for rare, urgent findings should also be considered depending upon the nature of the research method. Minors (or their legal representative) should be made aware of the planned timing of disclosure during the informed consent process.

- To whom should the results be communicated?

It should be made clear during the informed consent process to whom the individual results and IF will be communicated. Thus, depending of cases, the communication of individual results and IF could be directed to either the parents of the child, a designated health professional or an authorized third party (Beskow \& Burke 2010). When possible, researchers should therefore seek, and respect preferences of parents or legal guardians. When the research project involves adolescents, the researcher should, with parental permission, return results in a manner appropriate to their level of development, comprehension, and maturity. This possibility should be considered in the original consent discussion. For certain individual results and IF, it could be important to first divulge sensitive information to the minor in accordance with age and maturity.

\section{DISCUSSION}

The strength of these recommendations lies in the meaningful input from the systematic literature searches, the iterative process of stakeholder engagement and consultation, and targeted investigation of both parental and researcher perceptions regarding return of results and IF in paediatric research. These steps have ensured the RMGA Statement reflects a robust set of recommendations pursuant to current scientific knowledge and normative ethics. It is furthermore a living document, amenable to revision with the evolution of new technologies and ethical thought. 
Our efforts were limited in part due to inconsistent nomenclature used to describe studies on the return of results and IF. While we encouraged an iterative process of stakeholder input and revision, many of our empirical studies used hypothetical scenarios. As a result, we cannot yet report on barriers to implementation and uptake of the recommendations in practice. This remains the subject of future research in addition to the development of specific policies for paediatric biobanks and longitudinal studies, to name a few. While we recommend the researcher not be responsible for disclosure beyond the duration of the project, the rapidly changing interpretation of genomic results will challenge this.

\section{CONCLUSION}

The RMGA Statement provides guidance regarding when, and under what circumstances to return individual research results and IF discovered in pediatric genomics research. In order to protect and respect the integrity of each child, these recommendations are rooted in ethical principles and harmonized with existing paediatric best practices (Fabsitz et al. 2010). We expect these recommendations will help researchers navigate the complex ethical territory of returning IF in paediatric research. Areas of future research include exploring the barriers and facilitators to the uptake of these recommendations, and the experiences of patients and their families upon receiving IF of immediate versus future clinical import.

\section{ACKNOWLEDGMENTS}

The authors wish to thank the members of the Network of Applied Genetic Medicine of Québec (RMGA), FORGE (Finding of Rare Disease Genes) Canada Consortium), the Québec Association of Orphan Diseases, and Canadian Organization of Rare Disorders (CORD) for their comments and suggestions during the development of the RMGA Statement. 


\section{FUNDING}

This research was supported by the Canadian Institutes of Health Research and the Terry Fox Foundation (Grant No. TFF-105266); the Ministère de l'Économie, de l'Innovation et des Exportations du Québec (PSR-SIIRI-850); the Canadian Institute of Health Research and Genome Canada under the grant FORGE and CPCGC; Genome Canada under the Large Scale Applied Research Grant program for the project Orphan Diseases: Identifying Genes and Novel Therapeutics to Enhance Treatment (IGNITE) The Network of Applied Genetic Medicine of Québec (RMGA) (and the Fonds de recherche du Québec - Santé (FRQS) and Genome Atlantic.

\section{REFERENCES}

Abdul-Karim, R. et al., 2013. Disclosure of incidental findings from next-generation sequencing in pediatric genomic research. Pediatrics, 131(3): 564-71. doi 10.1542/peds.2012-0084

About CORD | Canadian Organization for Rare Disorders. Available at: https://www.raredisorders.ca/about-cord/ [Accessed October 31, 2015a].

Avard, D. et al., 2011. Pediatric research and the return of individual research results. $J$ Law, Med \& Ethics, 39(4): 593-604. doi 10.1111/j.1748-720X.2011.00626.x

Beskow, L.M. \& Burke, W., 2010. Offering individual genetic research results: context matters. Science Transl Med, 2(38): 38cm20. 10.1126/scitranslmed.3000952

Boycott, K. et al., 2015. The clinical application of genome-wide sequencing for monogenic diseases in Canada: Position Statement of the Canadian College of Medical Geneticists. $J$ Med Genet 52(7): 431-7. doi 10.1136/jmedgenet-2015-103144

Bredenoord, A.L. et al., 2011. Disclosure of individual genetic data to research participants: the 
debate reconsidered. Trends in Genetics, 27(2): 41-47. doi 10.1016/j.tig.2010.11.004

Burke, W. et al., 2013. Recommendations for returning genomic incidental findings? We need to talk! Genet Med, 15(11): 854-9. doi 10.1038/gim.2013.113

Canadian Institutes of Health Research Natural Sciences and Engineering Research Council of Canada and Social Sciences and Humanities Research Council of Canada, 2014. The Tri Council Policy Statement: Ethical Conduct for Research Involving Humans,

Canadian Institutes of Health Research, Natural Sciences and Engineering Research Council of Canada, and Social Sciences and Humanities Research Council of Canada, 2010. TriCouncil Policy Statement: Ethical Conduct of Research Involving humans,

Clayton, E.W., 2013. Returning Research Results of Pediatric Genomic Research to Participants.

Clayton, E.W. \& McGuire, A.L., 2012. The legal risks of returning results of genomics research. Genet Med, 14(4): 473-7. doi 10.1038/gim.2012.10

Davis, D.S., 2009. The parental investment factor and the child's right to an open future. The Hastings Center report, 39(2): 24-7.

Evans, J.P., 2013. Return of results to the families of children in genomic sequencing: tallying risks and benefits. Genet Med, 15(6) : 435-6. doi 10.1038/gim.2013.54

Fabsitz, R.R. et al., 2010. Ethical and practical guidelines for reporting genetic research results to study participants: updated guidelines from a National Heart, Lung, and Blood Institute working group. Circ Cardiovasc. Genet, 3(6): 574-80. doi 10.1161/CIRCGENETICS.110.958827

Fernandez, C. \& Canadian Paediatric Society Bioethics Committee, 2008. Ethical issues in health research in children | Position statements and practice points | Canadian Paediatric Society. 
Pediatric \& Child Health, 13(8): 707-712.

Fernandez, C. V et al., 2013. Attitudes of Canadian researchers toward the return to participants of incidental and targeted genomic findings obtained in a pediatric research setting. Genet Med, 15(7): 558-64. doi 10.1038/gim.2012.183

Fernandez, C. V et al., 2014. Attitudes of parents toward the return of targeted and incidental genomic research findings in children. Genet Med, 16(8): 633-40. doi 10.1038/gim.2013.201

Green, R.C. et al., 2013. ACMG recommendations for reporting of incidental findings in clinical exome and genome sequencing. Genet Med, 15(7): pp.565-74. doi 10.1038/gim.2013.73

Sainte Justine UHC, Announcing an innovative partnership between Sainte-Justine UHC and Génome Québec Dawn of a new era for children: Introducing Canada’s first integrated clinical genomic centre in pediatrics.

http://www.genomequebec.com/DATA/NOUVELLE/144_en V Dawn_of_a_new_era_for_ children__Introducing_Canadas_first_integrated_clinical_genomic_centre_in_pediatrics.pd f

Kleiderman, E. et al., 2014. Returning incidental findings from genetic research to children: views of parents of children affected by rare diseases. Journal of Medical Ethics, 40(10): 691-6. doi 10.1136/medethics-2013-101648

Knoppers, B. et al., 2014. Return of whole-genome sequencing results in paediatric research: a statement of the P3G international paediatrics platform. Eur J Hum Genet, 22(1): doi 10.1038/ejhg.2013.176

McGuire, A.L. et al., 2013. Point-counterpoint. Ethics and genomic incidental findings. Science, 340(6136) : 1047-8. doi 10.1126/science.1240156 
Netzer, C. et al., 2009. New challenges for informed consent through whole genome array testing. J Med Genet, 46(7): 495-6. doi 10.1136/jmg.2009.068015

Programmes | Public Population Project in Genomics and Society. Available at: http://p3g.org/programmes [Accessed November 6, 2015b].

Quebec Network of Applied Genetic Medicine, Statement of Principles on the Return of Research Results and Incidental Findings.

Rahimzadeh, V. et al., 2014. To disclose, or not to disclose? Context matters. Euro J Hum Gen, 23(3) : 279-84. doi 10.1038/ejhg.2014.108

RQMO | Regroupement québécois des maladies orphelines. Available at: http://rqmo.org/wp/ [Accessed November 6, 2015c].

Sénécal, K. et al., 2015. Frontline ethical issues in childhood cancer genetic reserach. In D. Malkin, ed. The Hereditary Basis of Childhood Cancer. p. In Press.

United Nations Convention on the Rights of the Child., 1989

Wolf, S.M., Annas, G.J. \& Elias, S., 2013. Point-counterpoint. Patient autonomy and incidental findings in clinical genomics. Science, 340(6136): 1049-50. doi 10.1126/science.1239119

Wright, C. et al., 2011. Next steps in the sequence: The implications of whole genome sequencing for health in the UK. PHG Foundation.

\footnotetext{
i For the purposes of this RMGA Statement, the concept of "paediatric research" refers to research with minors, including children and adolescents unable to legally consent to research participation. These recommendations should complement provincial laws and the norms governing legal recognition of minors' consent.
} 